\title{
IDENTIFICACIÓN Y DETERMINACIÓN ESTRUCTURAL DE UN SESQUITERPENO DE LAS HOJAS DE Tessaria integrifolia RUIZ \& PAV. Y EVALUACIÓN DE SU ACTIVIDAD LEISHMANICIDA
}

Carmen R. Silva-Correa1,a, José L. Cruzado-Razco ${ }^{1, b}$, María V. González-Blas ${ }^{1, a}$, Juan M. García-Armas ${ }^{2, c}$, Segundo G. Ruiz-Reyes ${ }^{1, d}$, Víctor E. Villarreal-La Torre ${ }^{1, a}$, César D. Gamarra-Sánchez ${ }^{1, e}$

\begin{abstract}
RESUMEN
Objetivos. Identificar y determinar la estructura del fitoconstituyente de hojas de Tessaria integrifolia Ruiz \& Pav con efecto leishmanicida. Materiales y métodos. Se preparó un extracto fluido de hojas de Tessaria integrifolia Ruiz \& Pav. que fue concentrado a extracto blando y se utilizó para evaluar la actividad leishmanicida en Mesocricetus auratus con leishmaniasis experimental, administrando vía intramuscular la dosis de $250 \mathrm{mg} / \mathrm{kg}$ del extracto blando por 15 días. El extracto fue fraccionado en columna cromatográfica de $45 \mathrm{~cm}$, con un diámetro de $2,5 \mathrm{~cm}$ que contiene silicagel G-60, de 70-230 mesh (Sigma-Aldrich $®$ ), las nueve fracciones obtenidas fueron evaluadas sobre macrófagos infectados con Leishmania sp para determinar la fracción activa y aislar el compuesto activo, mediante separación, purificación y cristalización, siendo analizado por resonancia magnética nuclear (RMN) de ${ }^{1} \mathrm{H},{ }^{13} \mathrm{C}$, y cromatografía líquida acoplada a espectroscopía de masas (LC/MS). Resultados. El extracto fluido de las hojas de Tessaria integrifolia Ruiz \& Pav. presenta actividad leishmanicida en Mesocricetus auratus y la fracción F8 es activa sobre macrófagos infectados a dosis de $14 \mu \mathrm{g} / \mathrm{mL}$. Se elucidó en esa fracción un sesquiterpeno tipo eudesmano ((4aS, 5R,6R,8aR)-6-hidroxi-5,8a-dimetil-3(1-metiletiliden) octahidronaftalen-2(1H)-ona), según análisis de $R M N{ }^{1} \mathrm{H},{ }^{13} \mathrm{C}, y \mathrm{LC} / \mathrm{MS}$. Conclusiones. El extracto fluido de hojas de Tessaria integrifolia Ruiz \& Pav. presenta actividad leishmanicida sobre Mesocricetus auratus. La fracción F8 presenta actividad leishmanicida sobre macrófagos infectados a dosis de $14 \mu \mathrm{g} / \mathrm{mL}$. Se elucidó en la fracción activa un sesquiterpeno tipo eudesmano según los análisis de $R M N{ }^{1} \mathrm{H},{ }^{13} \mathrm{C}$, y LC/MS
\end{abstract}

Palabras clave: Leishmania; Asteraceae; Sesquiterpenos de eudesmano; Mesocricetus (Fuente: DeCS BIREME).

\section{IDENTIFICATION AND STRUCTURAL DETERMINATION OF A SESQUITERPENE OF Tessaria integrifolia RUIZ \& PAV. LEAVES AND EVALUATION OF ITS LEISHMANICIDAL ACTIVITY}

\begin{abstract}
Objective. To identify and determine the phytoconstituent structure of Tessaria integrifolia Ruiz \& Pav. leaves with leishmanicidal activity. Materials and Methods. Fluid extract of leaves was prepared, concentrated to soft extract, and used to evaluate leishmanicidal activity in Mesocricetus auratus with experimental leishmaniasis, at the dose of $250 \mathrm{mg} /$ $\mathrm{kg}$ of soft extract by intramuscular route for 15 days. Extract was fractionated in $45 \mathrm{~cm}$ column chromatography with a $2.5 \mathrm{~cm}$ diameter, containing G-60 silica gel, and 70-230 mesh (Sigma-Aldrich $®$ ). Nine fractions were obtained and assessed on macrophages infected with Leishmania sp to determine the active fraction and isolate the active compound, by separation, purification, and crystallization, analyzed by nuclear magnetic resonance (NMR) of $1 \mathrm{H}, 13 \mathrm{C}$, and liquid chromatography coupled to mass spectroscopy (LC/ MS). Results. Fluid extract from the leaves of T. integrifolia presents leishmanicidal activity in M. auratus. Fraction F8 is active on infected macrophages at a dose of $14 \mu \mathrm{g} / \mathrm{mL}$. An eudesman type sesquiterpene ((4aS, 5R, 6R, 8aR) -6-hydroxy-5, 8a-dimethyl-3- (1-methylethylidene) octahydronaphthalen-2 (1H) one) was identified, by RMN $1 \mathrm{H}, 13 \mathrm{C}$, and LC / MS analysis. Conclusions. Fluid extract of leaves of Tessaria integrifolia Ruiz \& Pav. presents leishmanicidal activity on Mesocricetus auratus with experimental leishmaniasis. Fraction F8 presents leishmanicidal activity on infected macrophages at a dose of $14 \mu \mathrm{g} / \mathrm{mL}$. An eudesman type sesquiterpene was identified, according to $1 \mathrm{H}, 13 \mathrm{C}$, and LC / MS NMR analysis.
\end{abstract}

Keywords: Leishmania; Asteraceae; Eudesmane sesquiterpene; Mesocricetus (source: MeSH NLM).

\footnotetext{
Facultad de Farmacia y Bioquímica, Universidad Nacional de Trujillo. Trujillo, Perú.

Facultad de Ciencias de la Salud, Universidad Privada Antenor Orrego. Trujillo, Perú.

a Químico farmacéutico, magíster en Ciencias Químicas; b químico farmacéutico, magíster en Fisiología y Biofísica; ${ }^{c}$ ingeniero químico, doctor en Ciencias

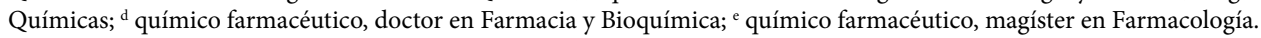

Recibido: 26/09/2017 Aprobado: 28/03/2018 En línea: 25/06/2018
} 


\section{INTRODUCCIÓN}

La leishmaniasis es una enfermedad parasitaria que se encuentra en unos 89 países y afecta entre 12 y 15 millones de personas en el mundo. Se estima que cada año se producen hasta dos millones de nuevos casos y 70000 muertes por la enfermedad (1). Asimismo, como enfermedad tropical es la segunda endemia en el Perú, y la tercera causa de morbilidad luego de la malaria y la tuberculosis ${ }^{(2)}$.

La leishmaniasis es causada por un protozoo del género Leishmania, que cuenta con más de 20 especies diferentes y se transmite a los humanos por la picadura de mosquitos hembras del género Lutzomyia. Clínicamente, la enfermedad se divide en tres formas principales, cutánea $(\mathrm{CL})$ conocida como uta, mucocutánea (MCL) y visceral (VL) conocida como Kala-azar ${ }^{(3)}$. Como primera línea de tratamiento se emplean los compuestos antimoniales pentavalentes que incluyen gluconato de antimonio sódico, antimoniato de meglumina y estibogluconato de sodio ${ }^{(4)}$.

Recientemente, el número de investigaciones sobre agentes leishmanicidas aumentó significativamente por dos razones: en primer lugar, por los efectos tóxicos observados con la terapia convencional, y en segundo lugar, la resistencia farmacológica de varias especies de Leishmania ${ }^{(5)}$. Además, los medicamentos más comúnmente utilizados no eliminan completamente los parásitos de todas las personas infectadas ${ }^{(6)}$. Debido a las limitaciones de los actuales esquemas terapéuticos, junto con la ausencia de vacunas adecuadas ${ }^{(7)}$, existe un interés creciente en nuevas sustancias con propiedades leishmanicidas obtenidas de extractos naturales que sean fácilmente accesibles para las personas afectadas ${ }^{(8)}$.

La especie Tessaria integrifolia Ruiz \& Pav. conocida como "pájaro bobo», «huapariu», «tseco», perteneciente a la familia de las Asteraceae, se encuentra ampliamente distribuida desde el sur de América Central hasta América del Sur, incluyendo Panamá, Colombia, Venezuela, Paraguay, Brasil, Bolivia y Perú ${ }^{(9)}$. T. integrifolia es un arbusto de hasta diez metros de alto, que crece en la ribera de los ríos de la costa y selva del Perú. Dentro de uso tradicional, se menciona que es antiasmático, antipirético, antiinflamatorio, y diurético ${ }^{(10)}$.

Investigaciones sobre la composición fitoquímica de $T$. integrifolia aislaron flavonoides como casticina y artemisina en las partes aéreas de la especie vegetal ${ }^{(11)}$. Asimismo, se reporta la presencia de flavonoides, lignanos, ácido cafeólico, eudesmanos sesquiterpénicos en el extracto metanólico de $T$. integrifolia aislando 23 compuestos diversos, entre ellos flavonoides como crisolfenol-D, quercetina, hiperina, y trifolina, incluye lignanos como pinoresinol y el 3,4-di(4hidroxi-3-metoxibencil) tetrahidrofurano, así como también derivados del ácido cafeólico ${ }^{(12)}$, sesquiterpenos de tipo eudesmanos, integrifósido $A$, integrifósido $B$, integrifósido $C$, integrifósido $D$ e integrifonol $A$ del extracto metanólico de la parte aérea de esta planta ${ }^{(13)}$. Otro estudio fitoquímico del

\section{MENSAJES CLAVE}

Motivación para realizar el estudio. La leishmaniasis es una enfermedad endémica que presenta una elevada incidencia mundial, los medicamentos no tienen la eficacia necesaria y producen importantes efectos adversos.

Principales hallazgos. La administración del extracto fluido de hojas de pájaro bobo presentó actividad leishmanicida en hámsters infectados experimentalmente.

Implicancias. La actividad leishmanicida del extracto fluido de hojas de pájaro bobo podría representar un beneficio potencial para el mejoramiento de la calidad de vida de la población afectada por esta enfermedad endémica.

extracto metanólico de las hojas y flores de $T$. integrifolia, informa la presencia de esteroides, flavonoides, compuestos fenólicos, además de saponinas en las hojas ${ }^{(14)}$. Por otro lado, estudios experimentales previos, determinaron que el extracto metanólico de las flores de $T$. integrifolia genera lisis, inmovilidad o falta de desarrollo de las promastigotas de Lesihmania ${ }^{(15,16)}$

En base a los antecedentes y considerando que existen pocos estudios realizados sobre los usos medicinales de esta especie vegetal en nuestro medio, se hace necesario evaluar la utilidad farmacológica de sus fitoconstituyentes sobre la leishmaniasis, al ser una enfermedad que se presenta endémicamente en áreas costeras, valles interandinos y selva del Perú.

\section{MATERIALES Y MÉTODOS}

\section{PREPARACIÓN DEL EXTRACTO}

Se recolectaron las hojas de $T$. integrifolia «pájaro bobo» en la ribera del río Chamán, ciudad de Chepén, provincia de Chepén, departamento de La Libertad (Altitud: $135 \mathrm{msnm}$, Latitud: $07^{\circ} 13^{\prime} 36^{\prime \prime}$, Longitud: $79^{\circ} 25^{\prime} 45^{\prime \prime}$ ). La planta medicinal se clasificó en el Herbarium Truxillense de la Universidad Nacional de Trujillo con el Código N 54937-HUT.

Se utilizó un kilogramo de hojas secas y molidas, se humectó con etanol $70^{\circ} \mathrm{GL}$, y se obtuvo por percolación un volumen equivalente a un litro de extracto fluido, el cual se concentró en evaporador rotativo Heidolph® a $37^{\circ} \mathrm{C}$ y 110 mbar de presión constante hasta obtención de extracto seco $(235 \mathrm{~g})$ con un rendimiento de $23,5 \%$.

\section{INFECCIÓN EXPERIMENTAL CON LEISHMANIASIS}

La muestra de Leishmania sp. fue aislada de un paciente voluntario procedente del distrito de Salpo, provincia de Otuzco, departamento de La Libertad, e inoculada directamente en tubos con cultivo Schneider suplementado con $10 \%$ de suero fetal bovino (SFB) (inactivado a $56^{\circ} \mathrm{C}$, 
durante 30 minutos). Como antimicrobiano se empleó gentamicina ( $80 \mathrm{mg} / 2 \mathrm{~mL}$ ) y se incubó por diez días a $25^{\circ} \mathrm{C}$. Se realizó recuento para determinar la concentración de promastigotas en el cultivo ${ }^{(17)}$.

Se emplearon 20 ejemplares machos de $M$. auratus de tres meses de edad con un peso promedio de $50 \mathrm{~g}$, adquiridos en el bioterio de la Facultad de Farmacia y Bioquímica de la Universidad Nacional de Trujillo. Los ejemplares fueron infectados con inóculo de 500 promastigotas $/ \mathrm{mL}$, aplicando la inyección intradérmica en el dorso de la nariz ${ }^{(18)}$, previa desinfección del área de la piel utilizando alcohol yodado como antiséptico, y empleando material aséptico y jeringas descartables de forma individual. Se realizó un seguimiento que duró 60 días hasta la aparición de lesiones características de leishmaniasis y mediante frotis del aspirado de las lesiones utilizando coloración Wright y lectura microscópica a 100X, se procedió confirmar la enfermedad y distribuir los grupos experimentales.

El grupo control estuvo conformado por diez ejemplares infectados experimentalmente con Leishmania sp; a los que se les administró solución salina durante 15 días. Por otro lado, el grupo tratado incluyó diez ejemplares infectados experimentalmente con Leishmania sp.; a los que se les administró el extracto de T. integrifolia a la dosis de $250 \mathrm{mg} /$ $\mathrm{kg} /$ día vía intramuscular durante 15 días.

El seguimiento de la evolución del grupo tratado se realizó diariamente por control fotográfico, para comparar la evolución de las lesiones frente al grupo control. Se realizaron frotis con el exudado de las lesiones utilizando coloración Wright y lectura microscópica a 100X. El porcentaje de efectividad se obtuvo en base al número de animales con diagnóstico negativo y diagnóstico positivo postratamiento.

\section{AISLAMIENTO Y SEPARACIÓN DE LOS METABOLITOS SECUNDARIOS}

Se utilizó una columna cromatográfica de $45 \mathrm{~cm}$, con un diámetro de 2,5 cm, que contiene Silicagel G-60, de 70-230 mesh (Sigma-Aldrich®), usando como fase móvil: hexanoacetato de etilo (2:3). Las fracciones cromatográficas se unieron de acuerdo a la similitud de sus cromatogramas y se realizó mediante cromatografía en capa fina (TLC) con placas de aluminio de Silicagel $60 \mathrm{~F}_{254}$ (Merck) y fase móvil: hexano-acetato de etilo (1:1). Finalmente se obtuvieron nueve fracciones: F1 (4,1 g), F2 (2,1 g), F3 (1,9 g), F4 (0,8 g), F5 (3,6 g), F6 (4,2 g), F7 (3,4 g), F8 (2,3 g) y F9 (5,5 g).

\section{EVALUACIÓN DE LA ACTIVIDAD LEISHMANICIDA IN} VITRO DE LAS FRACCIONES CROMATOGRÁFICAS

Se obtuvieron macrófagos de los ejemplares, administrando vía intraperitoneal (v.i.p.) $3 \mathrm{~mL}$ de solución de Hank's a las 24 horas y 48 horas; a las 72 horas se administró $5 \mathrm{~mL}$ de medio Waymouth v.i.p., diez minutos después se realizó el sacrificio de los animales de experimentación mediante asfixia con $\mathrm{CO}_{2}$. Se abrió asépticamente el área abdominal realizando incisión con el escalpelo para obtener mediante jeringas estériles el exudado peritoneal, que fue llevado directamente a las placas de cultivo celular que contenían medio Waymouth con $1 \mathrm{ml}$ de solución de gentamicina (40 mg) y se añadió la suspensión de cultivo de Leishmania sp. (200 promastigotas $/ \mathrm{mL}$ ). Seguidamente, las placas de cultivo fueron sometidas a un proceso de incubación a $25{ }^{\circ} \mathrm{C}$ con una atmósfera de $5 \%$ de $\mathrm{CO}_{2}$ durante 24 horas ${ }^{(19)}$, hasta la aparición de formas amastigotes dentro del macrófago. Luego los cultivos fueron expuestos a las fracciones cromatográficas a una concentración de $14 \mu \mathrm{g} /$ $\mathrm{mL}$ diluida en dimetilsulfóxido y cultivados a $25^{\circ} \mathrm{C}$ durante 24 horas. Los ensayos se realizaron por cuadriplicado para cada fracción cromatográfica y se compararon con un grupo control. Se realizó el conteo realizando frotis con coloración Wright y lectura microscópica a 100X, para evaluar la infección parasitaria en los macrófagos.

\section{ELUCIDACIÓN DEL COMPUESTO CON ACTIVIDAD LEISHMANICIDA}

Lo restante de la fracción cromatográfica activa F8 $(0,1974 \mathrm{~g})$ fue enviada a la Central Analítica del Instituto de Química de la Universidad de São Paulo (IQUSP) de Brasil para su purificación, cristalización y análisis mediante $\mathrm{RMN}{ }^{1} \mathrm{H}$ y ${ }^{13} \mathrm{C}$ en el equipo Bruker Alll $500 \mathrm{MHz}$, solvente: cloroformo deuterado $\left(\mathrm{CDCl}_{3}\right)$ y cromatografía líquida acoplada a espectroscopia de masa (MAxis 3G - Bruker Daltonics).

\section{ANÁLISIS ESTADÍSTICO}

La evaluación de los resultados obtenidos se realizó mediante la prueba de análisis de varianza (ANOVA) y la prueba de Tukey (HSD). Los análisis consideran un nivel de significancia del $95 \%$ y fueron realizados mediante el software Microsoft $₫$ Office Excel 2016 para Microsoft $\circledast$ Windows.

\section{ASPECTOS ÉTICOS}

El uso y manejo de los animales de experimentación se realizó de acuerdo a Guide for the Care and Use of Laboratory Animals ${ }^{(20)}$. La investigación fue aprobada por el Comité de Ética de la Universidad Nacional de Trujillo.

\section{RESULTADOS}

El extracto fluido de $T$. integrifolia presentó actividad leishmanicida a la dosis de $250 \mathrm{mg} / \mathrm{kg} /$ día en M. auratus con infección experimental de leishmaniasis, evidenciando disminución de las lesiones en el dorso de la nariz (Figura 1). La efectividad del tratamiento fue obtenida en relación al número de animales con diagnóstico negativo y positivo, posterior a los 15 días de tratamiento, observando 


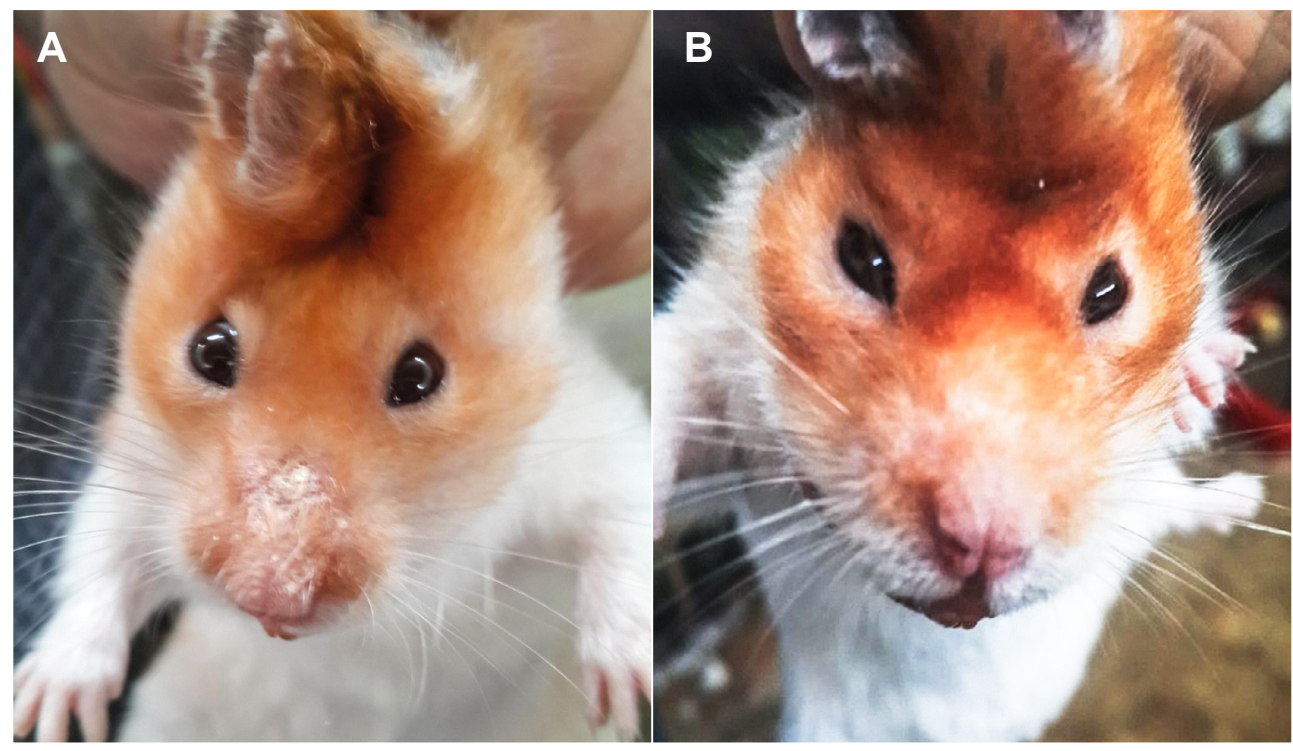

Figura 1. M. auratus del grupo tratado con T. integrifolia. A: Al iniciar el tratamiento. B: A los 15 días de tratamiento.

una efectividad del $95 \%$ en el grupo que recibió dosis del extracto en comparación al grupo control.

En la evaluación de la actividad leishmanicida de las nueve fracciones cromatográficas obtenidas del extracto fluido de las hojas de T. integrifolia, la fracción F8 demostró mayor actividad sobre macrófagos infectados a dosis de $14 \mu \mathrm{g} / \mathrm{mL}$ (Figura 2). El análisis estadístico ANOVA muestra diferencia significativa entre los grupos $(p<0,05)$ y según la prueba HSD de Tukey, hay diferencia significativa para la fracción F8 en relación a las demás fracciones.

El espectro de RMN de ${ }^{13} \mathrm{C}$ del compuesto aislado mostró señales para quince átomos de carbono y el espectro de RMN ${ }^{1} \mathrm{H}$ mostró señales para 24 protones (Tabla 1).

35

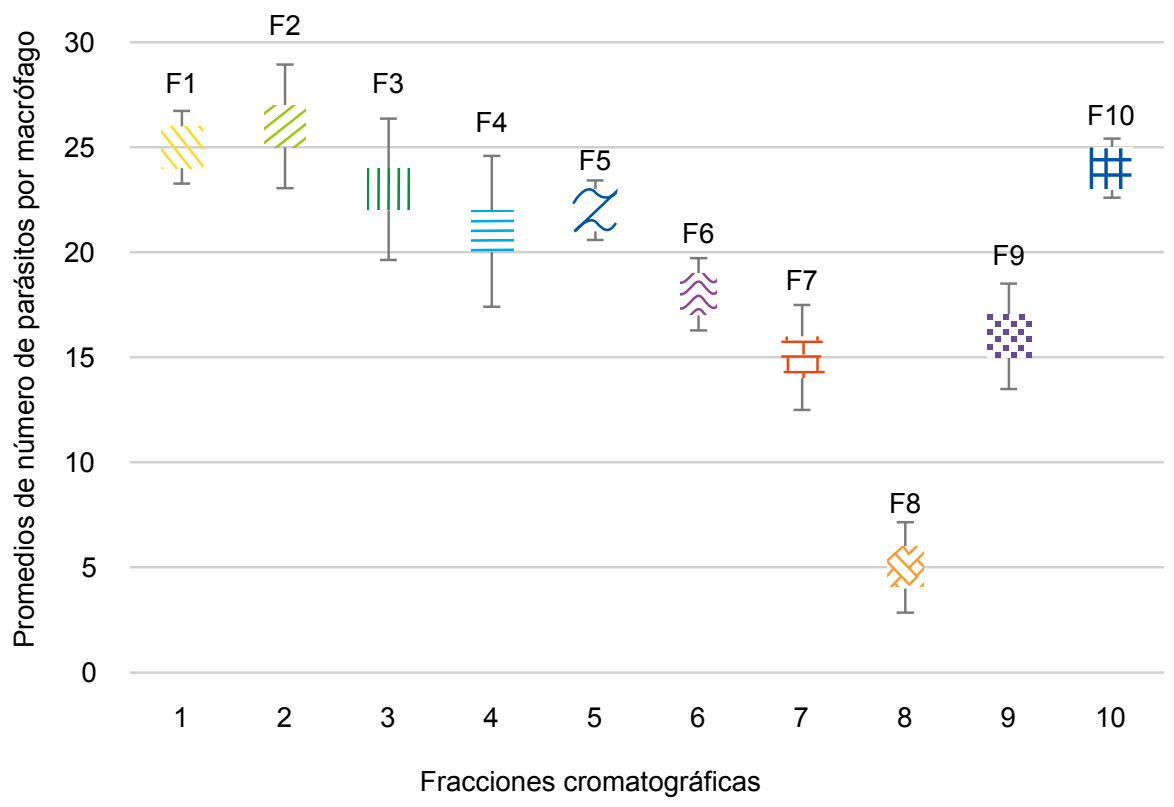

Figura 2. Actividad leishmanicida in vitro de las fracciones cromatográfícas de las hojas de T. integrifolia sobre macrófagos infectados. 
Tabla 1. Señales del espectro de RMN de ${ }^{1} \mathrm{H}$ y ${ }^{13} \mathrm{C}$ del compuesto aislado de la fracción $\mathrm{F} 8$ de las hojas de $T$. integrifolia

\begin{tabular}{|c|c|c|c|c|c|}
\hline${ }^{1} \mathbf{H}$ & $\delta(p p m)$ & Multiplicidad & $\begin{array}{l}\text { Número de } \\
\text { hidrógenos }\end{array}$ & ${ }^{13} \mathrm{C}$ & $\delta(p p m)$ \\
\hline $\mathrm{H}-1$ & 2,29 & $d$ & 2 & $\mathrm{C}-1$ & 40,75 \\
\hline $\mathrm{H}-4$ & 2,54 & $\mathrm{~d}, \mathrm{t}$ & 2 & $\mathrm{C}-2$ & 203,14 \\
\hline $\mathrm{H}-5$ & 1,72 & $\mathrm{~m}$ & 1 & $C-3$ & 133,21 \\
\hline $\mathrm{H}-6$ & 3,93 & $\mathrm{~m}$ & 1 & C-4 & 27,45 \\
\hline $\mathrm{H}-7$ & 1,96 & $d, t$ & 2 & C-5 & 39,88 \\
\hline $\mathrm{H}-8$ & 1,06 & $\mathrm{t}$ & 2 & C-6 & 72,29 \\
\hline $\mathrm{H}-10$ & 1,77 & $\mathrm{~m}$ & 1 & $\mathrm{C}-7$ & 26,09 \\
\hline $\mathrm{H}-11$ & 0,98 & d & 3 & C-8 & 36,91 \\
\hline $\mathrm{H}-12$ & 0,91 & $\mathrm{~s}$ & 3 & C-9 & 38,98 \\
\hline $\mathrm{H}-14$ & 1,23 & $\mathrm{~s}$ & 3 & C-10 & 39,94 \\
\hline \multirow[t]{5}{*}{$\mathrm{H}-15$} & 1,20 & s & 3 & C-11 & 18,93 \\
\hline & & & & C-12 & 15,27 \\
\hline & & & & C-13 & 145,23 \\
\hline & & & & C-14 & 27,86 \\
\hline & & & & C-15 & 27,61 \\
\hline
\end{tabular}

Datos obtenidos a $500 \mathrm{MHz}$ en $\mathrm{CDCl}_{3}$. Central Analítica del Instituto de Química de la Universidad de São Paulo (IQUSP), Brasil.

ppm: partes por millón

La elucidación estructural del compuesto aislado de la fracción F8 (10 mg) dio como resultado un sesquiterpeno tipo eudesmano ((4aS, 5R,6R,8aR)-6-hidroxi-5,8a-dimetil-3-(1metiletiliden) octahidronaftalen-2(1H)-ona), cuya estructura se muestra en la Figura 3, según los análisis resultantes de RMN $1 \mathrm{H}, 13 \mathrm{C}$ y LC/MS.

\section{DISCUSIÓN}

La evaluación leishmanicida in vivo se realizó mediante la infección experimental en $M$. auratus, se observó que los especímenes del grupo control mostrando lesiones

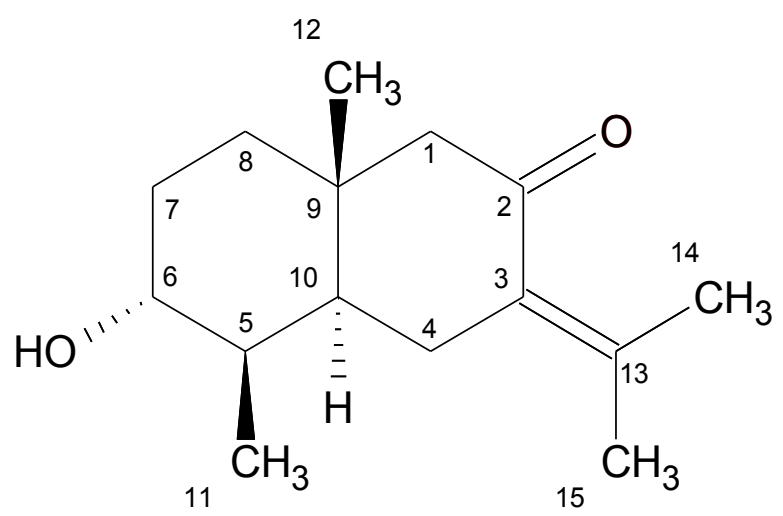

(4aS,5R,6R,8aR)-6-hidroxi-5,8a-dimetil-3-(1-metiletiliden) octahidronaftalen-2(1H)-ona

Figura 3. Propuesta de estructura del compuesto aislado de la fracción F8 de las hojas de T. integrifolia características de la infección en la zona del dorso de la nariz, donde se le inyectó promastigotas de Leishmania. Las lesiones fueron evidentes en los especímenes a los 60 días posteriores a la infección experimental. Los especímenes del grupo tratado que recibieron el tratamiento con el extracto de $T$. integrifolia a dosis de $250 \mathrm{mg} / \mathrm{kg}$ vía intramuscular durante 15 días, observándose una mejoría de las lesiones en el dorso de la nariz de los hámsters al término del tratamiento.

La efectividad del tratamiento fue obtenida en relación al número de animales con diagnóstico negativo y con diagnóstico positivo postratamientos de 15 días, observando una efectividad del $95 \%$ en el grupo tratado que recibió dosis del extracto en comparación al grupo control. En la evaluación leishmanicida in vivo se utilizó al $M$. auratus por ser altamente susceptible a varias especies de Leishmania, además se ha reportado que la susceptibilidad de los hámsters, es principalmente en los machos (21), presentándose incremento de citoquinas a nivel intracelular como la IL-4, IL-10 y el TGF- $\beta{ }^{(22)}$ como respuesta inmune de tipo mixta, que se observa en los diferentes lugares de infección. Los lugares anatómicos para la inoculación en los hámsteres son la cara dorsal del tarso, la base de la cola (región lumbosacra), la nariz (vía intradérmica) y el cojinete plantar (23).

La fracción F8 se purificó con solventes orgánicos hasta la obtención de cristales de color blanco en forma de agujas (10 mg), que fueron analizados mediante RMN de ${ }^{1} \mathrm{H} y{ }^{13} \mathrm{C} y$ cromatografía líquida acoplada a espectroscopia de masas (LC/MS) obteniendo un solo compuesto puro de esta 
fracción. El espectro de masas presenta la especie $\left[\mathrm{M}^{+1}\right]$ de $237,19 \mathrm{~m} / \mathrm{z}$, el análisis del fraccionamiento de la molécula corrobora la propuesta de estructura que corresponde a la fórmula $\mathrm{C}_{15} \mathrm{H}_{24} \mathrm{O}_{2}$.

De todo lo anterior, se infiere la presencia de al menos un componente biológicamente activo en $T$. integrifolia, que no ha sido reportado en trabajos anteriores con la misma especie, como el de Ono et al. que reporta el aislamiento y elucidación estructural de cinco compuestos eudesmanos, denominados integrifósidos A, B, C, D e integrifonol $A^{(13)}$, así también, Wu et al. realizaron una revisión de las estructuras y actividades biológicas de 972 sesquiterpenoides de tipo eudesmano de 247 especies (de 110 géneros) de la familia Asteraceae, en el cual no se reportó el compuesto aislado ${ }^{(24)}$. Al respecto, los sesquiterpenos de tipo eudesmano han mostrado actividad leishmanicida, como lo reporta la investigación realizada por García et al. donde evalúa el extracto etanólico y hexánico de hojas de la especie Pluchea carolinensis, atribuyendo a este tipo de estructuras eudesmanos como posibles responsables de la actividad leishmanicida ${ }^{(25)}$.

Hussain et al. realizaron una revisión de múltiples compuestos con actividad leishmanicida, de los cuales destacan los terpenos, algunos de ellos extraídos de Cistus monspeliensis y Cistus creticus que han mostrado muy buena actividad ${ }^{(26)}$. De estas sustancias extraídas, la de mayor interés con esta investigación, por su similitud estructural, es el óxido ent-3ß-acetoxi-13-epi-manoil (ribenol), estructura también reconocida por Chan et al. ${ }^{(27)}$ junto con Loukaci et al. ${ }^{(28)}$ que mencionan que tanto ribenol como sus derivados óxido ent-manoil presentan actividad contra promastigotes y amastigotes de Leishmania donovani. Además, cabe resaltar que Chan et al. adicionan que los derivados modificados de ribenol presentan mayor actividad que la misma. Ribenol muestra gran similitud estructural con la estructura aislada, tal y como se puede observar en la Figura 4, es por ello que planteamos que el mecanismo de actividad de esta última sea muy similar o igual al de ribenol.

Entre las principales limitaciones del estudio, tenemos que el compuesto aislado no pudo ser ensayado posteriormente en los modelos leishmanicidas in vitro e in vivo, debido a la concentración insuficiente que quedó luego de realizados los análisis de RMN de ${ }^{1} \mathrm{H}$ y ${ }^{13} \mathrm{C}$ en Brasil. Además, en este trabajo se utilizó cultivos de Leishmania sp aislados de pacientes, por lo que también hubiera sido importante,

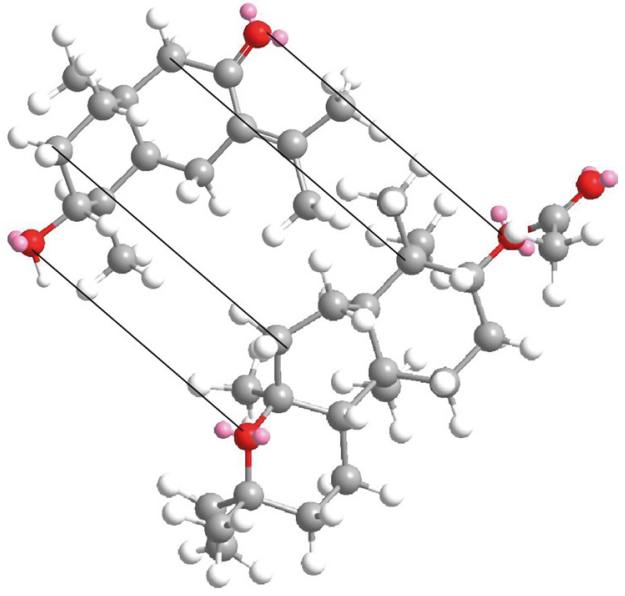

Figura 4. Similitud estructural entre la molécula aislada y ribenol.

evaluar la actividad leishmanicida en cultivos estándar de diversas especies de Leishmania, esto último no afecta la validez de los resultados, pero deja una base para continuar con esta investigación y complementarla.

Se concluye que el extracto fluido de hojas de $T$. integrifolia presenta actividad leishmanicida en $M$. auratus infectados experimentalmente a la dosis de $250 \mathrm{mg} / \mathrm{kg} / \mathrm{día}$ vía intramuscular durante 15 días. Se determinó que la fracción F8 del extracto fluido de las hojas de T. integrifolia presenta actividad leishmanicida sobre macrófagos infectados a dosis de $14 \mu \mathrm{g} / \mathrm{mL}$. Se elucidó de esta fracción F8 un compuesto sesquiterpeno de tipo eudesmano, según los resultados del análisis de resonancia magnética nuclear (RMN) de ${ }^{1} \mathrm{H},{ }^{13} \mathrm{C}$, y cromatografía líquida acoplada a espectroscopia de masa (LC/MS).

Agradecimientos: A la Dra. María S. Aurora Prado, docente de la Facultad de Farmacia de la Universidad de São Paulo por su apoyo con los análisis de $\mathrm{RMN}{ }^{1} \mathrm{H}$ y ${ }^{13} \mathrm{C}$.

Contribuciones de los autores: CS, JL, MG, JG, SG, VV y CG participaron en la concepción y diseño del artículo. MG, SG, CG obtuvieron financiamiento. CS y JC escribieron la primera versión del manuscrito. CS, JG y VV participaron en el análisis e interpretación de los datos. Todos los autores revisaron en forma crítica las versiones del manuscrito y aprobaron su versión final.

Fuentes de financiamiento: autofinanciado.

Conflicto de intereses: los autores declaran no tener conflictos de interés.

\section{REFERENCIAS BIBLIOGRÁFICAS}

1. Torres-Guerrero E, Quintanilla-Cedillo MR, Ruiz-Esmenjaud J, Arenas R. Leishmaniasis: a review. F1000Research. 2017;6:750

2. Espinoza-Morales D, Lucchetti Rodríguez A, Silva-Caso W, Suarez-Ognio L, Pons
MJ, Del Valle Mendoza J. An atypical case of disseminated cutaneous leishmaniasis due to Leishmania peruviana in the valleys of Ancash-Peru. Asian Pacific Asian Pac J Trop Med. 2017;10(11):1101-03.
3. Hashiguchia Y, Gomez E, Cáceres AG, Veleza LN, Villegas NV, et al. Andean cutaneous leishmaniasis (Andean-CL, uta) in Peru and Ecuador: the vector Lutzomyia sand flies and reservoir mammals. Acta Trop. 2018;178:264-75. 
4. Malerich J, Li J, Joder D, Vieria E, Keesen T, et al. Antileishmanial Activity of Natural Product-Like Naphthoquinones. Drug Dev. Res. 2013;74:237-41.

5. Bouyahya A, Et-Touys A, Dakka N, Fellah $\mathrm{H}$, Abrini J, Bakri Y. Antileishmanial potential of medicinal plant extracts from the North- West of Morocco. Beni-Suef Univ. J. Basic Appl. Sci. 2018;7:50-4.

6. de Menezes J, Guedes CE, Petersen AL, Fraga DB, Veras PS. Advances in Development of New Treatment for Leishmaniasis. Biomed Res Int. 2015;815023:11.

7. Monzote L, Piñón A, Setzer WN. Antileishmanial Potential of Tropical Rainforest Plant Extracts. Medicines. 2014;1,32-55.

8. Da Silva BJ, Souza-Monteiro JR, Rogez H, Crespo-López ME, Do Nascimento JL, Silva EO. Selective effects of Euterpe oleracea (açai) on Leishmania (Leishmania) amazonensis and Leishmania infantum. Biomed Pharmacother. 2018;97:16131621.

9. Mostacero J, Mejía F, Gamarra O. Fanerogamas del Perú. Taxonomía, utilidad y ecogeografia. la ed. Lima: CONCYTEC; 2009.p. 892

10. Mostacero J, Castillo F, Mejía F, Gamarra O, Charcape J, Ramirez R. Plantas Medicinales del Perú. Taxonomía, Ecogeografía, Fenología y Etnobotánica. 1a ed. Lima: Asamblea Nacional de Rectores; 2011. p. 604-605

11. Guerreiro E, Pestchanker M, Vitto L, Giordano O. Sesquiterpenes and flavonoids from Tessaria species. Phytochemistry. 1990;29(3):877-9.

12. Ono M, Masuoka C, Odake Y, IkegashiraS, Ito Y,Nohara T. Antioxidative Constituents from Tessaria integrifolia. Food Sci Technol Res. 2000;6(2):106-14.

13. Ono M, Masuoka C, Odake Y, Ito Y, Nohara T. Eudesmane derivatives from
Tessaria integrifolia. Phytochemistry. 2000;53(4):479-84.

14. Pérez F, Rodríguez F, Sagástegui A. Estudio fitoquímico preliminar de Tessaria integrifolia R. et P. Cienc. Salud. 2007;1(1).2-5.

15. Marín E, Pérez F, Sagástegui A. Actividad antileishmaniásica in vitro del extracto Metanólico de las flores de Tessaria integrifolia $\mathrm{R}$ et P. (Asteraceae). Pueblo Cont. 2007;18(1):101-8.

16. Caballero J. Evaluación fitoquímica $y$ actividad antimicrobiana de Tessaria integrifolia, recurso medicinal de Perú. [Tesis Doctoral]. Trujillo: Escuela de Posgrado, Universidad Nacional de Trujillo; 2014.

17. Cruzado L, Silva C, Rodríguez A, Escajadillo H. Aislamiento de Leishmania sp. en lesión perianal en una paciente procedente del distrito de Sanagorán. provincia Sánchez Carrión. Pharmaciencia. 2013;1(1):24-7.

18. Comandolli C, Grafova I, Naiff M, Avella M, Gentile G, Grafov A, Franco A. Topical treatment of experimental cutaneous leishmaniasis in golden hamster (Mesocricetus auratus) with formulations containing pentamidine. Acta Amaz. 2017;47(1):39-46.

19. Arias G. Evaluación de cinco derivados de los benzimidazoles contra promastigotes metacíclicos y macrófagos infectados de $L$. mexicana. [Tesis de Maestría]. México D.F.: Instituto Politécnico Nacional; 2009.

20. National Research Council of the Academy of Sciences. Guide for the care and use of laboratory animals. 8a ed. Washington DC: The National Academies Press; 2011.

21. Travi BL, Osorio Y, Melby PC, Chandrasekar B, Arteaga L, Saravia N. Gender is a major determinant of the clinical evolution and immune response in hamsters infected with Leishmania sp. Infect Immun. 2002;70(5):2288-96.

22. Osorio Y, Melby PC, Pirmez C, Chandrasekar B, Guarin N, Travi BL. The site of cutaneous infection influences the immunological response and clinical outcome of hamsters infected with Leishmania panamensis. Parasite Immunol. 2003;25(3):139-48.

23. Robledo S, Carrillo L, Daza A, Restrepo A, Muñoz D, et al. Cutaneous leishmaniasis in the dorsal skin of hamsters: a useful model for the screening of antileishmanial drugs. J Vis Exp. 2012;(62):e3533.

24. Wu QX, Shi YP, Jia ZJ. Eudesmane sesquiterpenoids from the Asteraceae family. Nat Prod Rep. 2006;23(5):699-734.

25. García M, Perera WH, Scull R, Monzote L. Antileishmanial assessment of leaf extracts from Pluchea carolinensis, Pluchea odorata and Pluchea rosea. Asian Pac J Trop Med. 2011;4(10):836-40.

26. Hussain $\mathrm{H}$, Al-Harrasi A, Al-Rawahi A, Green IR, Gibbons S. Fruitful decade for antileishmanial compounds from 2002 to late2011.Chem Rev.2014;114(20):10369428.

27. Chan-Bacab MJ, Peña-Rodriguez LM. Plant natural products with leishmanicidal activity. Nat Prod Rep. 2001;18(6):674-88.

28. Loukaci A, Kayser O, Bindseil K, Siems K, Frevert J, Abreu PM. New Trichothecenes Isolated from Holarrhena floribunda. J Nat Prod. 2000;63(1):52-6.

Correspondencia: Carmen Rosa Silva Correa

Dirección: Calle Moscú $N^{\circ} 331$, Urbanización

San Nicolás, Trujillo, Perú

Teléfono: (+51)964112623

Correoelectrónico: csilva@unitru.edu.pe 